\title{
ChiNing Decoction
}

National Cancer Institute

\section{Source}

National Cancer Institute. ChiNing Decoction. NCI Thesaurus. Code C119741.

A decoction of Liang Ge San, a traditional Chinese herbal medicine, with potential antiinflammatory and anti-stomatitis activities. Although the complete mechanism of action through which the ChiNing decoction works has yet to be fully elucidated, upon oral administration, the active ingredients may inhibit the inflammatory response, possibly by reducing the levels of pro-inflammatory cytokines, such as interleukin 6 (IL-6) and tumor necrosis factor-alpha (TNFa), in the saliva. This may protect the oral mucosa against these inflammatory mediators, and may reduce and relieve radiation-induced stomatitis and the associated pain. 